\title{
Observational properties of relativistic black hole winds
}

\author{
Ken-ya Watarai $\dagger$ \\ Department of Education, Osaka Kyoiku University, 582-8582, Osaka, Japan \\ email: watarai@cc.osaka-kyoiku.ac.jp
}

\begin{abstract}
We examine observational properties of relativistic black hole winds as an origin of high luminosity sources such as microquasars and ultra-luminous X-ray sources (ULXs). When strong relativistic wind/outflow happens in the vicinity of the black hole, the wind might form the optically-thick photosphere. Therefore the emission observed in ULXs might come from the photosphere of the wind, not from the accretion disk.

We found that the location of the photosphere is larger than the disk thickness for superEddington mass-outflow rates and sub-relativistic wind velocities $(v \sim 0.1-0.2 c)$. To understand the radiative structure in the high luminosity sources, we should take into account not only the emission from the accretion disk but also the emission from the outflow at the same time.
\end{abstract}

Keywords. Black hole physics - stars: winds, outflows - X-rays: binaries

\section{Introduction}

As for observed thermal spectral components in Galactic black hole candidates, it generally seems that accretion disk is the origin. However, if strong, radiation-pressure driven wind blows in a luminous black hole candidate, how do we observe the wind? King \& Pounds (2003) have recently proposed the black hole wind scenario in order to explain the origin of the high luminosity in ULXs and a few PG quasars. Here we will discuss the observational properties using a simple relativistic wind model for a stellar mass black hole.

\section{Model for Relativistic Black Hole Winds}

Our model for relativistic black hole wind is based on Abramowicz et al. (1991). Assumptions of the model are steady, spherical symmetry, special/general relativity. From mass conservation equation, the density distribution in co-moving frame is given by

$$
\rho=\frac{\dot{M}_{0}}{4 \pi R^{2} v Y^{*}},
$$

where $\dot{M}_{0}$ is the mass-outflow rate, and $v$ is the wind velocity, and $R$ is the distance from a black hole, $R=\sqrt{r^{2}+z^{2}}$, respectively. The relativistic corrections are included in $Y^{*}=\gamma g_{00}^{1 / 2}$, where $\gamma=\left(1-\beta^{2}\right)^{-1 / 2}$, and $g_{00}=\left(1-r_{\mathrm{g}} / R\right)$. Here $\beta$ is the wind velocity normalized by the speed of light, $\beta \equiv v / c$, and $r_{\mathrm{g}}$ is the Schwarzschild radius, $r_{\mathrm{g}}=2 G M / c^{2}$. We assume that the wind velocity $\beta$ is constant, and a 10 solar mass black hole.

$\dagger$ Research fellow of the Japan Society for the Promotion of Science. 

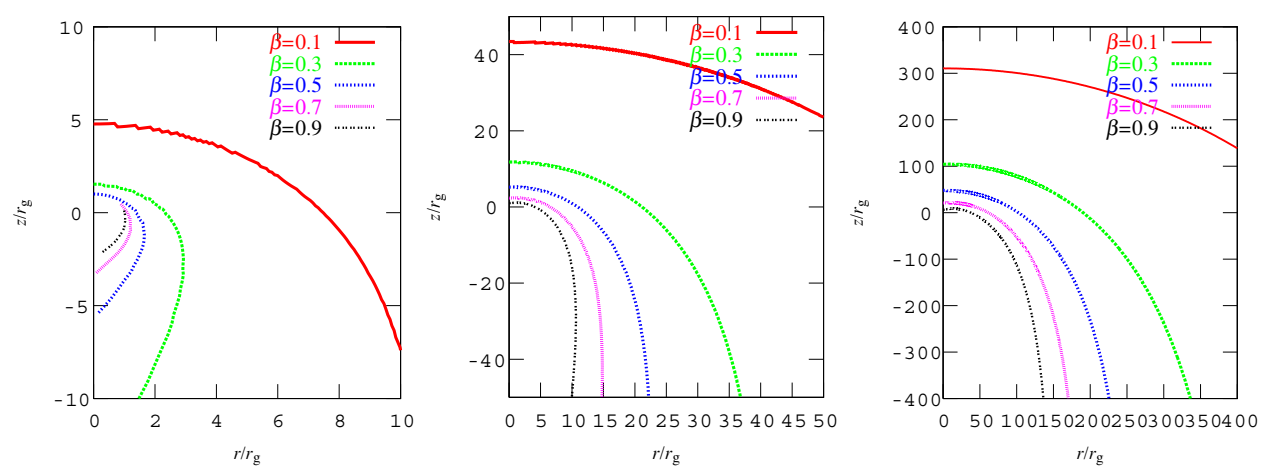

Figure 1. Location of the photosphere for various wind velocities $\beta$. Mass loss rates, $\dot{m}_{0}=\dot{M}_{0} / \dot{M}_{\text {Edd }}$, equal to 1,10 , and 100 from left to right.

\section{Calculation Method \& Location of the Photosphere}

To calculate flux around a Schwarzschild black hole, we adopt the "Ray-Tracing Method" including special/general relativity (Luminet 1979). Total optical depth of moving media from an observer at infinity to the location of the "photosphere" is given by

$$
\tau_{\mathrm{ph}}=\int_{R_{\mathrm{ph}}}^{\infty} \kappa \rho \gamma(1-\beta \cos \theta) d s=1
$$

where $\theta, \kappa, d s$ are the viewing angle to the observer, opacity for electron scattering, and small distance along the null geodesics. We obtain the location of the photosphere, $R_{\mathrm{ph}}$, from the $\tau_{\mathrm{ph}}=1$ condition. The location of the photosphere is shown in figure 1 .

Increasing of $\dot{m}_{0}(\gtrsim 10)$ leads to high-density wind, and the $R_{\mathrm{ph}}$ also be large. On the other hand, when the wind velocity closes to the light speed for high- $\dot{m}_{0}$, the density of the wind decreases because of the mass conservation. Thus we obtain small emitting region. The $R_{\mathrm{ph}}$ is given by the combination of these parameters. We note that the location of the photosphere is larger than the disk thickness in high- $\dot{m}_{0}$ and low- $\beta$ cases.

\section{Conclusions}

According to Begelman et al. (2006), mass-loss rate in SS433 is extremely high, $d M / d t=5 \times 10^{-4} M_{\odot} y r^{-1}$. In this surroundings we can no longer see the inner accretion disk, the emission from the photosphere of the outflow will be observed. As for ULXs, if the super-Eddington mass outflow occurs, the size of the photosphere increases, but the temperature of the outflow decreases as increasing $R_{\mathrm{ph}}$. Thus this feature conflicts with the observations in several ULXs. Comparing time-dependent spectral properties with various models will be important in the future, and it will help to understand real nature of ULXs.

\section{Acknowledgements}

This work was supported in part by the Grants-in-Aid of the Ministry of Education, Science, Sports, and Culture of Japan (16004706; K.W.).

\section{References}

Abramowicz, M. A., Novikov, I. D. \& Paczyński, B. 1991, ApJ, 369, 175

King, A. R. \& Pounds, K. A. 2003, MNRAS, 345, 657

Luminet, J.-P. 1979, A\&A, 75, 228 